\title{
ULTRAMICROELISA PARA LA DETECCIÓN DE ANTICUERPOS IgM ANTI M. leprae
}

\author{
José LAFERTE(1), EIba G. ABREU(1), René ROBAINA(2) \& Vicente VEREZ(3)
}

\begin{abstract}
RESUMEN
La disponibilidad del sistema Ultramicroanalítico (SUMA) y de un antígeno especieespecífico del M. leprae obtenido mediante síntesis química, permitió la normalización y validación de un ultramicroELISA para la detección de anticuerpos IgM específicos a esta micobacteria. El análisis de 433 sueros de banco de sangre y 265 sueros usados para validar el método y clasificados en un grupo control de donantes de banco de sangre (100), un grupo de pacientes tuberculosos (50), un grupo de enfermos de lepra (65) y un grupo de contactos de estos enfermos (50), mostró la especificidad del ensayo para evidenciar la infección con el M. leprae.
\end{abstract}

Los resultados obtenidos del estudio adicional de 140 muestras de suero de contactos de enfermos estuvieron estrechamente correlacionados $(r=0,98)$ con los resultados obtenidos por la técnica de microELISA convencional.

La utilización del SUMA no solo permite un notable ahorro de reactivos si no además facilita la lectura, cálculo, validación y almacenamiento automático de los resultados.

UNITERMOS: UltramicroELISA

\section{INTRODUCCION}

La lepra es una infección crónica causada por un parásito intracelular obligado: el Mycobacterium leprae, esto determina que los mecanismos de defensa en las personas infectadas sean fundamentalmente de tipo celular y no de tipo humoral. A pesar de esto se ha observado una correspondencia entre la carga bacilar y el nivel de anticuerpos presentes en un individuo infectado, detectandose generalmente niveles considerables de anticuerpos en las formas multibacilares de la enfermedad y niveles más bajos en las formas paucibacilares ${ }^{14}$.

Estudios realizados recientemente han demostrado la validez de los ensayos dirigidos a detectar anticuerpos específicos al M. leprae, lo que representa una valiosa ayuda en la identificación temprana de personas infectadas, en alto riesgo de enfermar y quizás también de individuos infectados que aún no presentan signos clínicos y constituyen diseminadores silenciosos de la enfermedad. La localización precoz de estos individuos es importante para lograr el corte de la cadena de transmisión $n^{5,6}$. Una contribución importante a la realización práctica de estos ensayos lo constituyó el aislamiento y la caracterización de un antígeno especie-específico del M. leprae: el glicolípido fenólico $\mathrm{I}^{1 \mathrm{1}, 12}$ cuya utilización en la técnica de ELISA demostró la presencia de anticuerpos (fundamentalmente de tipo IgM) al mismo en pacientes con lepra ${ }^{1,7,15,16}$.

El análisis de los determinantes antigénicos de esto glicolípido utilizando anticuerpos poli y monoclonales, mostró la inmunodominancia del residuo terminal ${ }^{3,6}$ di-o-metil glucosa $a^{11,17}$ y estos elementos sirvieron de base para la elaboración de antígenos sintéticos, los cuales acoplados a una proteina portadora (seroalbúmina bovina) ofrecieron similares resultados que con el antígeno natu-

(1) Instituto de Medicina Tropical "Pedro Kouri". Apdo. 601 Siboney, Ciudad Habana, Cuba.

(2) Centro de Inmunoensayo. Apdo. 6945. Ciudad Habana, Cuba.

(3) Laboratorio de Química de los Carbohidratos. Universidad de la Habana. Facultad de Química. Ciudad Habana, Cuba.

Dirección para correspondencia: Lic. José Laferté. Instituto de Medicina Tropical “Pedro Kourí”. Apdo. 601. Siboney, Ciudad de la Habana, Cuba. 
LAFERTE, J.; ABREU, E.G.; ROBAINA, R. \& VEREZ, V. - UltramicroELISA para la detección de anticuerpos IgM anti M. leprae. Rev. Inst. Med. trop. S. Paulo, 33 (6): 491-495, 1991.

ral en la técnica de microELISA ${ }^{4,8,9,10,13}$.

En este trabajo se presentan los resultados obtenidos en la normalización y validación de un ultramicroelisa para la detección de anticuerpos IgM específicos al M. leprae, que puede constituir una valiosa ayuda para el pesquisaje masivo en las áreas endémicas y como instrumento para el control de la enfermedad.

\section{MATERIALES Y METODOS}

\section{Reactivos}

Antígeno. Fue obtenido sintéticamente ${ }^{13}$ y donado por el laboratorio de química de los carbohidratos de la Universidad de la Habana, Cuba.

Conjugado. Para su preparación se utilizó un antisuero, anti IgM humana monoespecífico que fue donado por el Centro de inmunoensayo Ciudad de la Habana, Cuba. La fracción IgG de este antisuero se obtuvo mediante un proceso de purificación de la fracción gamma obtenida por precipitación salina y fue entonces utilizada para la conjugación con la enzima beta galactosidasa recombinante de $\mathrm{E}$. coli, según el método del glutaraldehido en un paso ${ }^{2}$. Esta enzima fue suministrada por el centro de Ingenieria Genética y Biotecnologia Ciudad de la Habana, Cuba.

\section{Sustrato}

Se utilizó el 4 metillumbelliferyl beta $D$ galactopiranósido (sustrato fluorigénico) que fue suministrado por el laboratorio de química de los Carbohidratos de la Universidad de la Habana, Cuba. Como solución fluorescente de referencia se empleó la 4 metilumbelliferona obtenida de la Koch Light Limited, Harvehill, Suffolk, England.

Los demás reactivos químicos empleados en las soluciones tampón comúnes fueron de la $\mathrm{BDH}$.

\section{Muestras}

Se procesaron un total de 838 muestras de sueros, el grupo control (533 sueros) fue obtenido de donantes de un banco de sangre de Ciudad de la Habana. Los sueros del grupo de pacientes tuberculosos (50) fueron obtenidos en el hospital antituberculoso de Ciudad de la Habana, los sueros de enfermos de lepra (65) y de contactos de enfermos (190) fueron donados por el Instituto de Medicina Tropical "Pedro Kourî" y el Centro Provincial de Higiene y Epidemiologia de Guantánamo, Cuba.

\section{Equipos}

Fue utilizado un equipo SUMA modelo 121-B consistente en un espectrofluorímetro-nefelómetro automático acoplado a una microcomputadora y una multipipeta de 96 posiciones computarizada (ERIZO 101). Se utilizaron además otros accesorios específicos para el procesamiento de muestras y reactivos en el rango ultramicroanalítico. Centro de Inmunoensayo. Ciudad de la Habana, Cuba.

\section{UltramicroELISA}

Las placas PVC-SUMA de 96 cavidades fueron sensibilizadas con el antígeno a una concentración de $2 \mu \mathrm{g} / \mathrm{ml}$ en tampón carbonato-bicarbonato pH 9.6. La conservación de las mismas se realizó a $4^{\circ} \mathrm{C}$ hasta el momento de su utilización.

Las muestras y sueros controles se diluyeron 1:100 en tampón TRIS-Tween (TRIS-T) $15 \mathrm{mM}$, conteniendo suero de carnero al 5\%, se distribuyeron a razón de 10 microlitros por cavidad en la placa de reacción con el empleo de la multipipeta ERIZO 101 y se realizó la incubación durante 1 hora a $37^{\circ} \mathrm{C}$ en cámara humeda. $\mathrm{Al}$ término de este tiempo se procedió a realizar el lavado con tampón TRIS-T $15 \mathrm{mM}$ y posteriomente se adicionó el conjugado anti IgM humana betagalactosidasa, diluido 1:1600 en tampon TRIS-T $15 \mathrm{Mm}$, conteniendo suero de carnero al 5\%, se realizó la incubación durante 1 hora a $37^{\circ} \mathrm{C}$ y posteriormente el lavado en las mismas condiciones anteriormente descritas.

Con la ayuda de la multipipeta de 96 posiciones se dispensaron 10 microlitros de la solución de sustrato por cada cavidad de las placas de reacción y se incubaron durante 30 minutos a $37^{\circ} \mathrm{C}$. La fluorescencia resultante fue estimada en el espectrofluorímetro automático SUMA 121 y los resultados fueron expresados por la relación (F-B)/ (P-B), donde:

F: unidades de fluorescencia de la muestra. 
LAFERTE, J.; ABREU, E.G.; ROBAINA, R. \& VEREZ, V. - UltramicroELISA para la detección de anticuerpos IgM anti M. leprae. Rev. Inst. Med. trop. S. Paulo, 33 (6): 491-495, 1991.

P: unidades de fluorescencia del control positivo.

B: unidades de fluorescencia del blanco.

El criterio de seropositividad fue determinado previamente sobre la distribución de una población sana, sin contacto conocido con el M. leprae, utilizando el criterio del 98 percentil recomendado por la OMS. Los resultados obtenidos en la evaluación de la respuesta analítica de 433 muestras de suero de esta población, se ilustran en la Figura 1. Se seleccionó 0.135 como criterio de seropositividad del ensayo.

Figura 1. Frecuencia de distribución de las respuestas analiticas en 433 muestras de suero de una población normal. Se seleccionó 0.135 como criterio de seropositividad del ensayo ( 98 percentil).

\section{FRECUENCIA}

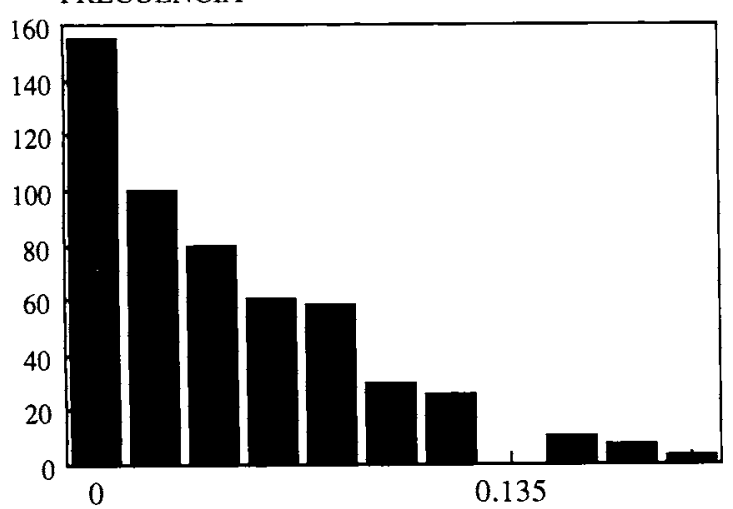

RELACION MUESTRA/CONTROL POSITIVO

\section{RESULTADOS Y DISCUSION}

Las concentraciones de los reactivos y las condiciones de la reacción fueron determinadas mediante titulación cruzada, seleccionandose aquellas que dieron los mejores resultados en el ensayo (Figuras 2-4). Para cada caso (antígeno de recubrimiento, dilución del suero y dilución del conjugado) se escogió aquel punto en la curva de titulación donde la relación positivo-negativo fue superior a 10 y el nivel de fondo fue bajo. En el caso particular del antígeno de recubrimiento se seleccionó la concentración de $2 \mu \mathrm{g} / \mathrm{ml}$ como la adecuada para el trabajo la cual corresponde al punto mínimo de saturación en la curva de titulación (Figura 2).
Figura 2. Titulacion del antigeno de recubrimiento. Se selleccionó la concentración de 2 microgramos por mililitro como la óptima para la sensibilización de las ultramicroplacas.

\section{FLUORESCENCIA (\%)}

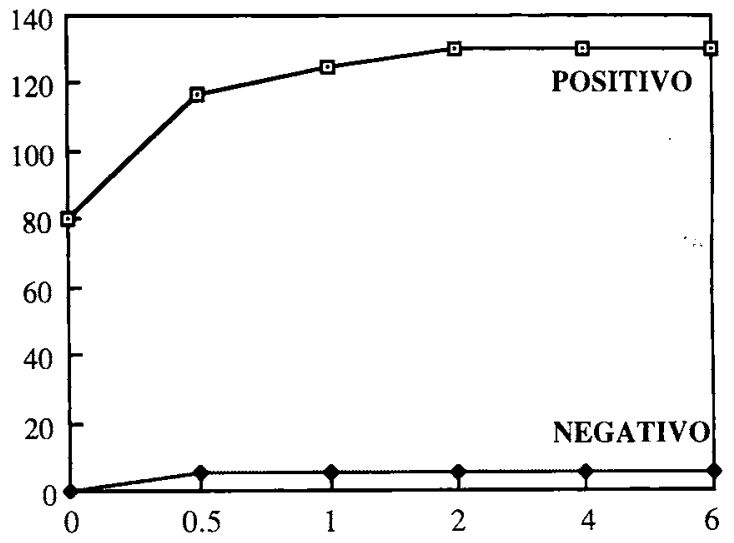

CONCENTRACIONES DE ANTIGENO (ug/ml)

Las placas de reacción sensibilizadas en estas condiciones fueron estables a $4^{\circ} \mathrm{C}$ durante un periodo de 12 meses, lo cual significó no solo un ahorro de tiempo y reactivos, sino además una disminución de la variabilidad de la técnica por este concepto.

Solo un $2 \%$ de los individuos del grupo control presentaron una respuesta que excedió al limite normal establecido para el ensayo (Tabla 1). A diferencia de esto los porcientos obtenidos en el grupo de enfermos fueron muy superiores, especificamente en el grupo afectado con las formas multibacilares de la enfermedad (93\%). En este grupo generalmente se detectan niveles elevados de anticuerpos IgM especificos al M. leprae ${ }^{16}$.

\section{TABLA 1.}

Resultados obtenidos en la detección de anticuerpos IgM al $\mathbf{M}$. leprae en grupo de sueros mediante el ensayo de ultramicroELISA.

\begin{tabular}{lrcr}
\hline & № & $>0.135$ & $\%$ \\
CONTROLES & 100 & 2 & 2 \\
TUBERCULOSOS & 50 & 0 & 0 \\
CONTACTOS & 50 & 12 & 24 \\
LEPRA PAUCIBACILAR & 25 & 8 & 32 \\
LEPRA MULTIBACILAR & 40 & 37 & 93 \\
\hline
\end{tabular}

No se observó reactividad cruzada en ninguno de los 50 sueros de pacientes tuberculosos ensaya- 
LAFERTE, J.; ABREU, E.G.; ROBAINA, R. \& VEREZ, V. - UltramicroELISA para la detección de anticuerpos IgM anti M. leprae. Rev. Inst. Med. trop. S. Paulo, 33 (6): 491-495, 1991.

Figura 3. Curvas de titulación de los sueros controles. La dilución 1:100 fue seleccionada para las muestras.

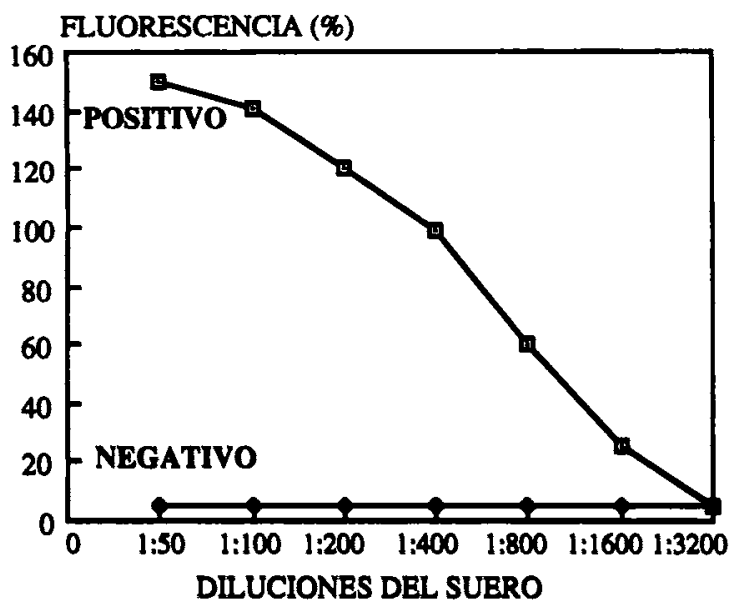

Figura 4. Curvas de titulación del conjugado anti IgM humana Bgalactosidasa. La dilución 1:1600 fue seleccionada para el ensayo.

\section{FLUORESCENCIA (\%)}

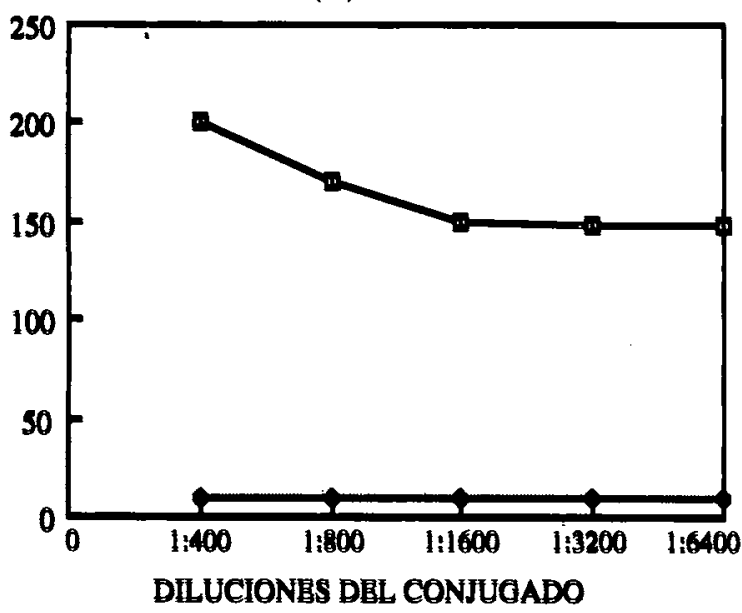

dos y el estudio de un grupo de contactos de enfermos de lepra reveló la presencia de anticuerpos IgM ant $M$. leprae en un nivel superior al grupo control. Esto podria ser de utilidad para la identificación de individuos presumiblemente infectados y con riesgo de enfermar en las áreas endémicas.

Las respuestas de 140 muestras de suero de contactos de enfermos de lepra, procesados paralelamente por microELISA y ultramicroBLISA (FIgura 5) mostraron un alto grado de correspondencia entre los resultados $(r=0.98)$.
Estudios posteriores podrian igualmente valorar la utilidad práctica de esta técnica en el monitoreo de pacientes bajo tratamiento, lo cual ha sido reportado para el sistema microELISA del mismo principio ${ }^{3}$.

Figura 5. Correlación del microELISA y del ultramicroELISA en la detección de anticuerpos IgM al $\mathbf{M}$. leprae en 140 muestras de suero de contactos de enfermos.

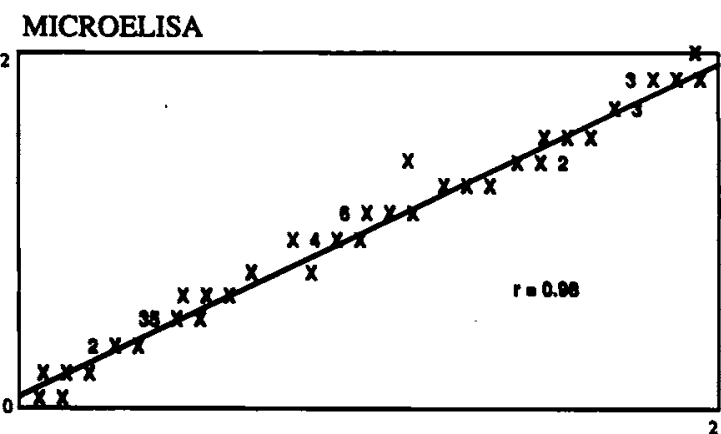

ULTRAMICROELISA

El Sistema Ultramicroanalítico (SUMA) ha sido usado con éxito en diferentes programas de control en Cuba (alfafetoproteína, hipotiroisdismo congénito, detección de anticuerpos al virus de la inmunodeficiencia humana, detección del antigeno carcinoembrionario, detección del antígeno de superficie del virus de la hepatitis B, etc), siendo adecuado para el procesamiento de un gran número de muestras y empleando solo de 5 a 10 microlitros como volumen de reacción. Se obtiene además un incremento de la sensibilidad debido al uso de un sustrato fluorigénico y la utilización de una microcomputadora acoplada al espectrofluorimetro facilita el procedimiento de lectura, cálculo, validación y almacenamiento automático de los resultados.

La utilización de esta tecnologia unida al ompleo de la enzima betagalactosidasa aportan aspectos novedosos a los ensayos serológicos relacionados con el M. leprae permitiendo además disponer de un sistema rápido y económico para emprender estudios masivos en las áreas endémicas.

\section{SUMMARY}

\section{UltramicroELISA Assay for the Detection of Human IgM Antibodles to $M$. leprae.}

The availability of an ultramicroanalitic system (SUMA) and specie-specific antigen of $M$. 
LAFERTE, J.; ABREU, E.G.; ROBAINA, R. \& VEREZ, V. - UltramicroELISA para la detección de anticuerpos IgM anti M. leprae. Rev. Inst. Med. trop. S. Paulo, 33 (6): 491-495, 1991.

leprae obtained by chemical synthesis, have made possible the standardization and validation of an ultramicroELISA assay for detecting specific human IgM antibodies to this mycobacterium. The specificity of this test to demonstrate the infection with M. leprae was corroborated through a screening of 433 blood bank serum samples and other 265 from diferent groups (100, control group, 50 tuberculosis patients, 65 leprosy patients, 50 from household). The results obtained in the aditional study of 140 household sero showed a high conrelation $(r=0.98)$ with the conventional microELISA method. The use of SUMA allows saving reagents and time since sample handling, plate reading, print out and storing the data are computer assisted.

\section{REFERENCLAS BIBLIOGRAFICAS}

1. AGUADO SANCHEZ, G.; MALIK, A.; TOUGNE, C.; LAMBERT, P.H. \& ENGERS, H.D. - Simplification and standarization of serodiagnostic tests for leprosy based on Phenolic Glycolipid-I (GP-I) antígen. Symposium on the immunology of leprosy. Leprosy Rev., 57 (Suppl. 2): 83$93,1986$.

2. AVRAMEAS, J. - Coupling of enzimes to proteins with glutaraldehyde. Immunochemistry, 6: 43-52, 1969.

3. BACH, M.A.; WALLACH, D.; FLAGEUL, B.; HOFFENBACH, A. \& COTTENOT, F. - Antibodies to phenolic glycolipid - 1 and to whole Mycobacterium leprae in leprosy patients: evolution during therapy. Int. J. Leprosy, 54: 256-267, 1986.

4. BRETT, S.J.; PAYNE, S.N.; GIGG, J.; BURGESS, P. \& GIGG, R. - Use of synthetic glycoconjugates containing the Mycobacterium leprae specific and immunodominant epitope of phenolic glycolipid $I$ in the serology of leprosy. Clin. exp. Immunol., 64: 476-483, 1986.

5. ABE, M.; MINAGAWA, F.; YOSHINO, Y.; OZAWA, T.; SAIKAWA, K. \& SAITO, T. - Fluorescent Leprosy Antibody Absorption (FLA-ABS) Test for Detecting Subclinical Infection with Mycobacterium leprae. J. Int. Leprosy, 48: 109-119, 1980.

6. BUCHANAN, T.M.; YOUNG, D.B.; MILLER, R.A. \& KHANOLKAR, S.R. - Serodiagnosis of infection with Mycobacterium ieprae Int. J. Leprosy, 51: 524-530, 1983.

7. CHO, S.N.; YANAGIHARA, D.L.; HUNTER, S.W.; GELBER, R.H. \& BRENNAN, P.J. - Serological specificity of phenolic glycolipid I from Mycobacterium leprae and use in serodiagnosis of leprosy. Infect. Immun., 41: 1077-1083, 1983
8. CHO, S.N.; FUJIWARA, T; HUNTER, S.W.; REA, T.H; GELBER, R.H. \& BRENNAN, PJ. - Use of an artificial antigen containing the 3,6-di-O-methyl-B-Dglucopyranosyl epitope for the serodiagnosis of leprosy. J. infect. Dis., 150: 31 1-322, 1984

9. FUIIWARA, T; HUNTER, S.W.; CHO, S.N.; ASPINALL, G.O. \& BRENNAN, PJ. - Chemical synthesis and serology of disaccharides and trisaccharides of phenolic glycolipid antigens from the leprosy bacillus and preparation of disaccharide protein conjugate for serodiagnosis of leprosy. Infect. Immun., 43: 245-252, 1984.

10. GIGG, J.; GIGG, R.; PAYNE, S. \& CONANT, R. - The allyl group for protection in carbohydrate chemistry. $17^{*}$ synthesis of propyl-O-(3,6-Di-O-methyl-B-Dglucopyranosyl)-( $1 \rightarrow 4)$-O-(2,3-Di-O-Methyl- $\infty-\mathrm{L}-$ rhamanopyranosyl)-(1 $\rightarrow$ 2)-3-O-methyl-L-rhamnopyranoside: the oligosaccharide portion of the major serologically active glucolipid from Mycobacterium leprae. Chem. Phys. Lip., 38: 299, 1985.

11. HUNTER, S.W. \& BRENNAN, P.J. - A novel phenolic glycolipid from Mycobacterium leprae possibly involved in immunogenecity and pathogenicity. J. Bact., 147: 728 735,1981 .

12. HUNTER, S.W.; FUJIWARA, T. \& BRENNAN, P.J. Structure and antigenicity of the major specific glycolipid antigen of Mycobacterium leprae J. biol. Chem., 257: $15072-15078,1982$.

13. MARIÑO, J.R.; BENCOMO, V.V.; GONZALEZ, L. \& PEREZ, C.S. - Synthesis of allyl and benzyl 4-0 (3,6 di-Omethyl-8-D) glucopiranosil 1-2, 3-Di-O methyl-L thamnopyranoside. Carbohydr. Res., 165: 197-206, 1987.

14. SANSONETTI, P.H. \& LAGRANGE, P.H. - The immunology of leprosy: speculations on the leprosy spectrum. Rev. infect. Dis., 3: 422-469, 1981.

15. YOUNG, D.B. \& BUCHANAN, T.M. - A serological test for leprosy with a glycolipid specific for Mycobacterium leprae. Science, 221: 1057-1059, 1983.

16. YOUNG, D.B.; DISSANAYAKE, S.; MILLER, R.A.; KHANOLKAR, S.R. \& BUCHANAN, T.M. - Humans respond predominantly with IgM immunoglobulin to the specie-specific glycolipid of Mycobacterium leprae. J. infect. Dis., 149: 870-873, 1984.

17. YOUNG, D.B.; KHANOLKAR, S.R.; BARG, L.L. \& BUCHANAN, T.M. - Generation and characterization of monoclonal antibodies to the phenolic glycolipid of Mycobacterium leprae. Infect. Immun., 43: 183-188, 1984.

Recebido para publicação em 16/05/1990. Aceito para publicação em 01/04/1991. 